ARTICLE

\title{
Aluminum depletion induced by co-segregation of carbon and boron in a bcc-iron grain boundary
}

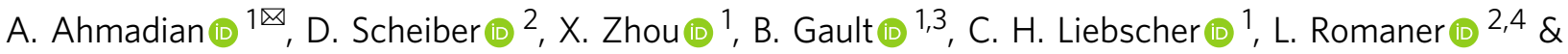 \\ G. Dehm (1) ${ }^{1}$
}

The local variation of grain boundary atomic structure and chemistry caused by segregation of impurities influences the macroscopic properties of polycrystalline materials. Here, the effect of co-segregation of carbon and boron on the depletion of aluminum at a $\Sigma 5(310)[001]$ tilt grain boundary in a $\alpha-\mathrm{Fe}-4$ at\% $\mathrm{Al}$ bicrystal is studied by combining atomic resolution scanning transmission electron microscopy, atom probe tomography and density functional theory calculations. The atomic grain boundary structural units mostly resemble kite-type motifs and the structure appears disrupted by atomic scale defects. Atom probe tomography reveals that carbon and boron impurities are co-segregating to the grain boundary reaching levels of $>1.5$ at\%, whereas aluminum is locally depleted by approx. 2 at.\%. First-principles calculations indicate that carbon and boron exhibit the strongest segregation tendency and their repulsive interaction with aluminum promotes its depletion from the grain boundary. It is also predicted that substitutional segregation of boron atoms may contribute to local distortions of the kite-type structural units. These results suggest that the co-segregation and interaction of interstitial impurities with substitutional solutes strongly influences grain boundary composition and with this the properties of the interface.

\footnotetext{
${ }^{1}$ Max-Planck-Institut fuer Eisenforschung GmbH, Düsseldorf, Germany. ${ }^{2}$ Materials Center Leoben GmbH, Leoben, Austria. ${ }^{3}$ Department of Materials, Royal School of Mines, Imperial College London, London, UK. ${ }^{4}$ Montanuniversität Leoben, Leoben, Austria. ${ }^{凶}$ email: a.ahmadian@mpie.de
} 
S egregation of solutes and impurity atoms to grain boundaries (GBs) have been studied extensively in the last decades because of their strong influence on the macroscopic physical and mechanical properties of materials ${ }^{1-10}$. Typically, GB segregation is studied in binary alloys serving as model systems to discern segregation tendencies of individual solutes ${ }^{1,4,9,10}$. However, technologically relevant alloy systems adopt a complex composition, which is tailored to maximize their mechanical properties. The interplay of different solutes in ternary or higher order systems on grain boundary segregation is far less understood ${ }^{11,12}$. Xing et al. ${ }^{12}$ developed a simple thermodynamic model to capture the competing mechanisms in ternary alloys that decrease, increase or do not affect GB segregation. However, this model has limited applicability when non-metallic elements are involved, which play a critical role in understanding embrittlement effects in iron-based alloys or steels ${ }^{3,13}$. Furthermore, co-segregation effects have also been observed to play an important role in other material systems such as ceramic and oxide materials ${ }^{14,15}$. Wang et al. ${ }^{15}$ observed complex co-segregation patterns of $\mathrm{Ca}$ and $\mathrm{Ti}$ at $\mathrm{MgO} \Sigma 5\left(\begin{array}{lll}3 & 1 & 0\end{array}\right)\left[\begin{array}{lll}0 & 0 & 1\end{array}\right] \mathrm{GBs}$ by atomic resolution imaging, which strongly affect the electronic structure of the GB and stabilize it.

Grain boundary segregation in iron $(\mathrm{Fe})$ is often characterized by Auger electron spectroscopy (AES) providing excellent chemical sensitivity, but limited spatial resolution leaving the structure of the GB elusive. However, it could be shown that sulfur (S) and phosphorous (P) are segregating to $\mathrm{GBs}^{16,17}$ in $\alpha-\mathrm{Fe}$ promoting embrittlement of the material. To prevent this detrimental tendency, carbon (C) or boron (B) can be introduced, which hinder the diffusion of $\mathrm{S}$ and $\mathrm{P}$ to GBs and therefore enhance their cohesive strength ${ }^{18-20}$.

The isolated role of $\mathrm{B}$ on increasing GB cohesion has long been anticipated in a range of material systems ${ }^{20-26}$ and it has been shown that the addition of small amounts of B partially suppresses intergranular fracture in brittle intermetallic FeAl alloys ${ }^{7,8,27}$. It is generally also assumed that $\mathrm{C}$ acts as a GB cohesion enhancer and recent ab-initio calculations found that the theoretical fracture strength of $\sum 5$ tilt GBs increases with increasing $C$ excess concentration $^{28-30}$.

Aluminum ( $\mathrm{Al}$ ) is an important alloying element in $\mathrm{Fe}$ and especially light weight steels, where a reduction in density on the order of $7.5 \%$ can be achieved by additions of up to $7 \mathrm{wt} \% \mathrm{Al}$ $(16 \text { at } \%)^{31}$. Hence, it is of great scientific and technological interest, but studies exploring the effect of $\mathrm{Al}$ on $\mathrm{GB}$ properties are rare ${ }^{32,33}$. Yuasa et al. ${ }^{32}$ concluded from density functional theory (DFT) calculations that $\mathrm{Al}$ grain boundary segregation will increase the GB energy and weaken the boundary strength due to a change from metallic to covalent-like bonding. However, Geng et al. ${ }^{33}$ showed a slight decrease in GB energy by $\mathrm{Al}$ addition through first-principal calculations, which is also in agreement with the Rice-Wang thermodynamic model ${ }^{34}$. It should be noted that only binary $\mathrm{Fe}-\mathrm{Al}$ systems were considered in the previously mentioned studies and insights into the impact of co-segregation effects are missing. For example, Rellick et al. ${ }^{35}$ concluded from fracture tests upon quenching that small quantities of $\mathrm{Al}$ would hinder the segregation of residual oxygen (O) to GBs, which impedes intergranular fracture. But the low $\mathrm{C}$ content could also be responsible for mitigating embrittlement and the role of $\mathrm{Al}$ on GB properties remains elusive, since the GB structure and composition could not be determined. Therefore, it is of great interest to investigate $\mathrm{GB}$ segregation of $\mathrm{Al}$ in $\mathrm{Fe}$ and to explore the impact of prominent impurity elements such as $\mathrm{C}$ and $\mathrm{B}$ on segregation thermodynamics since all three elements are common alloying elements in $\mathrm{Fe}$ and high performance steel.

Deciphering co-segregation phenomena requires a complete understanding of the atomic grain boundary structure and composition as well as description of the interaction between impurities ${ }^{13,36}$. The correlation of atom probe tomography (APT) and transmission electron microscopy (TEM) has paved the way to obtain insights into the segregation behavior of solutes even in GBs of complex alloys 37 . However, the underlying atomistic effects and the interplay of elements on the grain boundary segregation behavior are often uncharted.

The discrete atomic GB structure and the presence of defects at the GB are additional factors impacting the segregation behavior. It is known that $\mathrm{C}$ and $\mathrm{B}$ tend to segregate to sites of high stress fields such as dislocation cores ${ }^{38}$. Therefore it is indispensable to resolve the GB structure and composition at atomic resolution to relate the underlying segregation mechanisms by ab-initio calculations. For example, Medlin et al. ${ }^{39}$ investigated an asymmetric $\Sigma 5 \mathrm{~GB}$ in pure Fe using scanning transmission electron microscopy (STEM). They found that the GB adopts a complex topography by dissociating into nanofacets consisting of $\left(\begin{array}{lll}2 & 1 & 0\end{array}\right)$ and $\left(\begin{array}{lll}3 & 1 & 0\end{array}\right)$ facets, which are separated by GB facet junctions. However, connecting such complex GB structure and the segregation of minor impurities like S, P, C and B, which are even relevant in pure $\mathrm{Fe}$, is challenging by STEM alone. Besides using AES to study GB segregation in ferritic Fe and stee ${ }^{4,20,40}, \mathrm{APT}^{9,10}$ is capable of providing $3 \mathrm{D}$ compositional information with high elemental sensitivity. Aberrations arising from the field evaporation process ${ }^{41,42}$ limit the spatial resolution of APT effectively to approx. $0.5 \mathrm{~nm}^{43}$ for precipitates. For grain boundary structure and other crystalline defects, the spatial resolution is expected to be in a similar range or better ${ }^{44}$. Ultimately, the combination of atomic resolution STEM and APT provides structural as well as compositional information of the GB with the highest spatial and elemental resolution ${ }^{45-50}$.

In the present work, we correlate atomic resolution STEM, energy-dispersive X-ray spectroscopy (EDS) and APT measurements on a $\Sigma 5\left(\begin{array}{lll}3 & 1 & 0\end{array}\right)\left[\begin{array}{lll}0 & 0 & 1\end{array}\right]$ tilt $\mathrm{GB}$ in $\alpha-\mathrm{Fe}-4$ at $\% \mathrm{Al}$ to resolve the atomic GB structure and its local composition. The GB predominantly adopts a kite-type structural unit and the presence of $\mathrm{GB}$ defects is indicated. The $\mathrm{Al}$ concentration at the $\mathrm{GB}$ is observed to decrease, whereas the impurities $\mathrm{B}$ and $\mathrm{C}$ are clearly segregating. Complementary first-principles based DFT calculations are employed to elucidate the segregation tendencies of $\mathrm{Al}$, $\mathrm{B}$ and $\mathrm{C}$ and shed light onto the impact of solute interactions on the co-segregation behavior. A kinetic model is employed to investigate the temperature and time dependent segregation of solutes for similar conditions as in the experiment.

\section{Results}

Atomic GB structure. A low-magnification high angle annular dark-field (HAADF)-STEM image of the $\Sigma 5$ (3 10$)$ GB observed in $\left[\begin{array}{lll}0 & 0 & 1\end{array}\right]$ direction is shown in Fig. 1a. The local contrast in Fig. 1

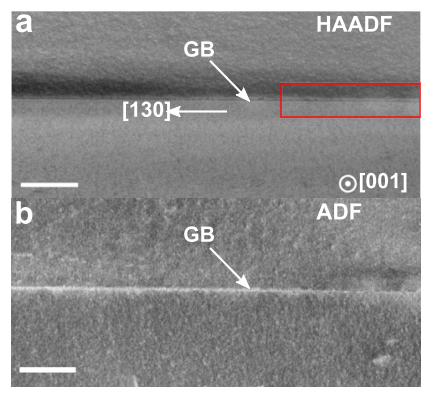

Fig. 1 Overview images of the $\Sigma 5\left(\begin{array}{lll}3 & 1 & 0\end{array}\right)\left[\begin{array}{lll}0 & 0 & 1\end{array}\right]$ GB. a HAADF-STEM image and (b) corresponding low-angle ADF-STEM image showing bright contrast at the GB core indicating an accumulation of strain fields at the GB. The red rectangle in (a) indicates regions, which were imaged at atomic resolution. The scale bar is $100 \mathrm{~nm}$. 


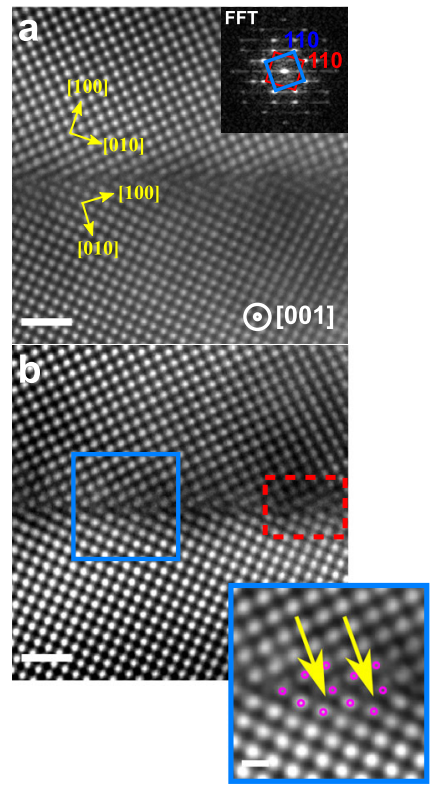

Fig. 2 Atomic structure of the $\Sigma 5\left(\begin{array}{lll}3 & 1 & 0\end{array}\right)\left[\begin{array}{lll}0 & 0 & 1\end{array}\right] \mathrm{GB}$ in the Fe-4at\% Al bi-crystal. a Raw HAADF-STEM image and corresponding FFT. From the FFT the misorientation angle is determined to $38^{\circ}$. b Fourier-filtered image of (a). The inset shows a magnified view of the kite-type structural GB units highlighted by magenta dots. The yellow arrows indicate positions where weak contrast in the kite centers is observed. The scale bar in (a and b) is $1 \mathrm{~nm}$, while the inset has a scale bar of $500 \mathrm{pm}$.

a is mainly attributed to changes in sample thickness and atomic mass. At a constant sample thickness the intensity is proportional to $Z^{1.5-2}$, with $Z$ being the atomic number ${ }^{51}$. At this length scale the GB appears straight and the formation of GB defects, such as steps or facets can not be resolved. The dark contrast of the GB may be attributed to GB grooving, a lower atomic density or lattice strain, causing dechannelling of the electron beam. Figure $1 \mathrm{~b}$ shows the simultaneously acquired low-angle ADF-STEM image revealing bright contrast along the GB, which may be attributed to the accumulation of strain fields at the $\mathrm{GB}^{46}$. The red square in Fig. 1 a indicates a segment of the boundary, which was imaged at atomic resolution with a local sample thickness of about $40 \mathrm{~nm}$ as determined by electron energy loss spectroscopy (EELS) measurements.

A representative atomic resolution HAADF-STEM image of the GB (Fig. 2)) shows the projected structural units of the $\Sigma 5\left(\begin{array}{lll}3 & 1 & 0\end{array}\right)\left[\begin{array}{lll}0 & 0 & 1\end{array}\right]$ GB viewed along the [0 0101$]$ direction. The blue and red squares in the corresponding fast Fourier transform (FFT) shown in the inset of Fig. 2a highlight the $<110>$ type reflections of the lower and upper grains, respectively. Both grains are misoriented by $\sim 38^{\circ}$ around the common [ $\left[\begin{array}{ll}0 & 0\end{array}\right]$ tilt axis. This observation is in good agreement with the EBSD analysis presented in Supplementary Fig. 1. A Butterworth Fourier-filter was applied to the image to remove low-frequency noise and highlight the atom columns at the boundary as shown in Fig. 2b. A magnified view reveals the kite-type structural units of the $\Sigma 5\left(\begin{array}{lll}3 & 1 & 0\end{array}\right)\left[\begin{array}{lll}0 & 0 & 1\end{array}\right] \mathrm{GB}$, which is consistent with the predicted structure of a pure bcc-Fe GB ${ }^{39,52}$. However, it should be noted, that a weak signal is apparent in the open regions of the kites (indicated by yellow arrows). In addition, the kite-structure of the GB is distorted on the right-hand side in Fig. $2 \mathrm{~b}$ (indicated by a red dotted rectangle). These features and their origins will be discussed later.

Besides kite-type structural units, defects such as steps (see Supplementary Fig. 2) or facets are present in other locations of

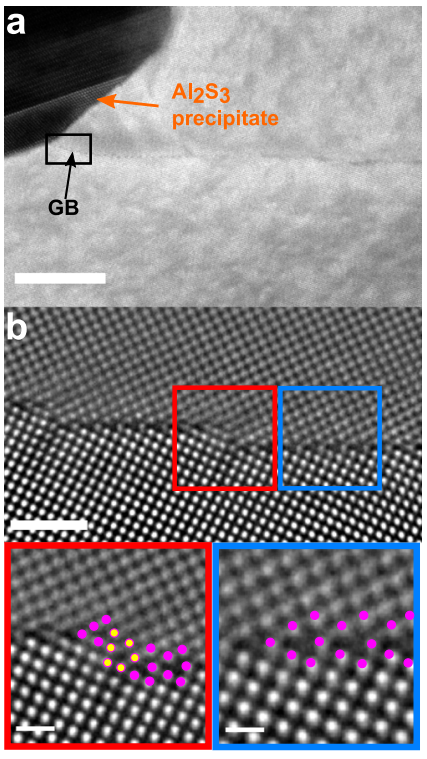

Fig. 3 Grain boundary nano-facetting in close vicinity to a GB precipitate. a HAADF-STEM image taken near the $\mathrm{Al}_{2} \mathrm{~S}_{3}$ precipitate. The black rectangle indicates region where the boundary is bend. $\mathbf{b}$ Atomic resolution image of the region within the black rectangle showing $\mathrm{nm}$ sized facets. The blue and red boxes indicate the symmetric facet and the step, respectively, as well as magnified views of the corresponding structural units. The scale bar is $20 \mathrm{~nm}$ in (a) and $1 \mathrm{~nm}$ in (b) and $500 \mathrm{pm}$ in the insets.

the GB, which are introduced due to a local change in GB inclination. Figure 3a shows a HAADF-STEM image of a GB segment intersecting an $\mathrm{Al}_{2} \mathrm{~S}_{3}$ precipitate. The local GB curvature is increased in close vicinity of the precipitate as indicated by a black rectangle in Fig. 3a. Atomic resolution imaging reveals a nano-facetted GB (Fig. 3b). It can be seen that while the lower grain is in [0 01 l] orientation, the upper grain is slightly off zone axis. The inclination angle of the GB with respect to the $\left(\begin{array}{lll}3 & 1 & 0\end{array}\right)$ plane is $\approx 7^{\circ}$ and the GB facets are adopting the symmetric $\Sigma 5$ (3 10 ) kite structure (blue box in Fig. 3b). The magnified view of a step (red box in Fig. 3b) reveals that it resembles a single kite structural unit, which is rotated by $\sim 87^{\circ}$ with a height of $\sim 0.46 \mathrm{~nm}$. It should be mentioned that in the investigated TEM specimen only one $\mathrm{Al}_{2} \mathrm{~S}_{3}$ precipitate (see Supplementary Fig. 3) was found, but even though a rather large GB curvature was introduced, the symmetric GB segments exhibit the kite-type structure.

Grain boundary segregation. In parts of the GB, the atomic arrangement has shown slight deviations form the perfect kite structure and the presence of a GB precipitates indicates that impurity segregation could impact the GB structure. Besides $\sim 4$ at $\% \mathrm{Al}$, the as-grown bicrystal contains different impurities such as $\mathrm{C}, \mathrm{B}, \mathrm{P}, \mathrm{S}$, and $\mathrm{Si}$ in the lower ppm range. Wet chemical analysis showed a bulk concentration of $C_{C} \approx 0.05$ at $\%$ and $C_{B} \approx 0.001$ at $\%$ for C and B (see Supplementary Table 2). STEM-EDS elemental mapping was used to determine the GB composition as shown in Fig. 4a. Here, the Al-K elemental map is superimposed on the simultaneously acquired HAADF-STEM image, where the dotted line highlights the position of the GB and the blue and red rectangles indicate regions where EDS spectra were integrated from. No Al enrichment at the GB is visible from the EDS map. To obtain a qualitative understanding of $\mathrm{Al}$ segregation, the spatial difference method was applied where the spectrum from within 
a
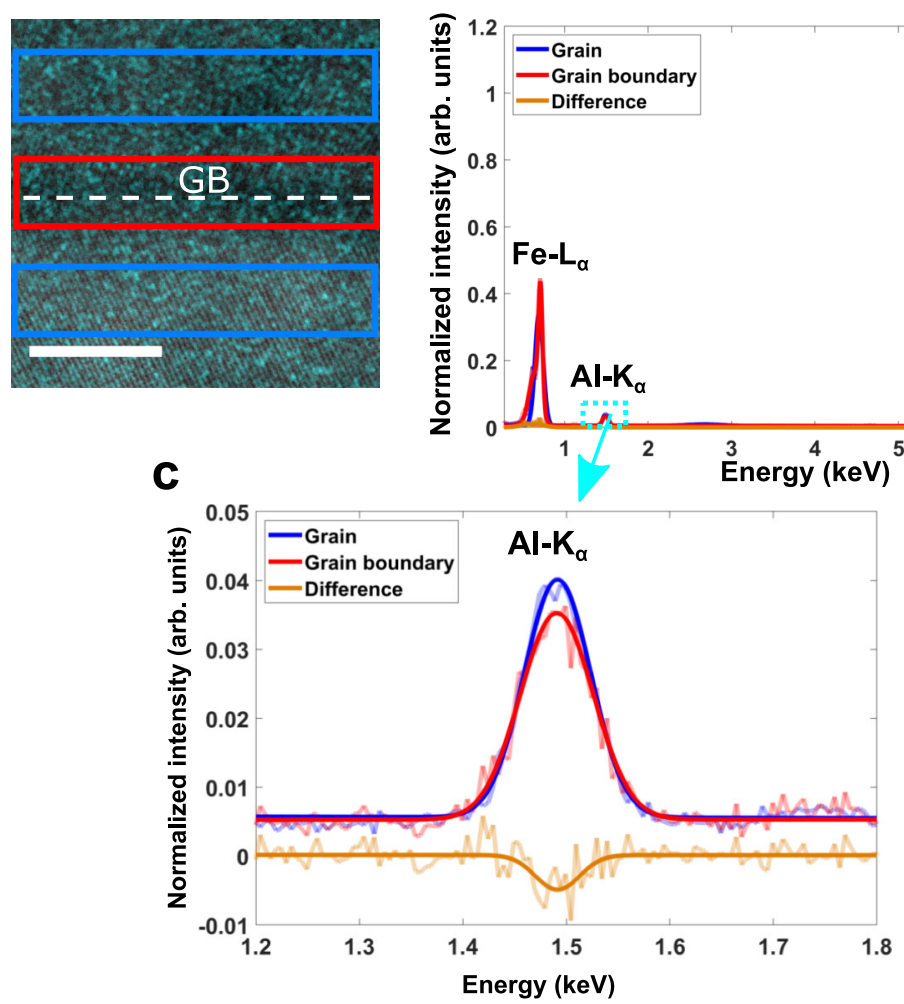

b

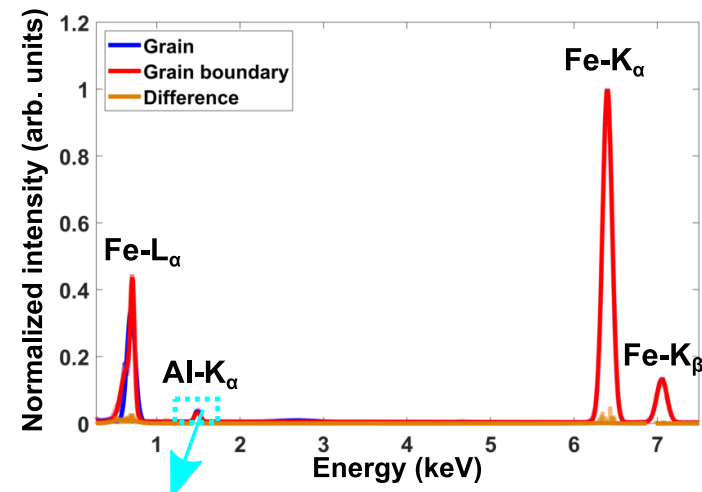

1.8

Fig. 4 Elemental distribution of $\mathbf{A l}$ at the $\mathbf{\Sigma} \mathbf{5}(\mathbf{3} \mathbf{1 0}) \mathbf{~ G B}$. a Overlay of the HAADF-STEM image and the Al-K elemental map taken across the $\Sigma 5(310)$ GB. The colored boxes indicate the location where the spectra in (b) were summed. b Summed EDS spectra, which were normalized to the Fe- $K_{\alpha}$ peak, extracted from the grain interior (blue) and the GB (red). The corresponding difference spectrum is shown in orange. In order to minimize effects from differences in peak shapes, the spectra were fitted by a Gaussian function. c A magnified view of the Al- $\mathrm{K}_{\alpha}$ edge indicates a slight decrease of Al at the GB. The semitransparent curves in ( $\mathbf{b}$ and $\mathbf{c}$ ) display the raw spectra, the solid lines the Gaussian fit. The Scale bar in (a) is $5 \mathrm{~nm}$.

the grains (blue) is subtracted from the GB spectrum (red). Each integration window had an area of $3 \times 10 \mathrm{~nm}^{2}$ in a region of constant thickness of $40 \mathrm{~nm}$ as proved by STEM-EELS. The two spectra of the grain interior were averaged and for comparison all spectra were normalized according to the $\mathrm{Fe}-\mathrm{K}_{\alpha}$ edge as illustrated in Fig. $4 \mathrm{~b}$. The difference spectrum (orange curve) has a non-zero value at the $\mathrm{Fe}-\mathrm{K}_{\alpha}$ edge, which is not caused by a slight shape difference of the peaks within the grain and at the GB. Therefore, all peaks in the spectra were fitted by Gaussian peaks (solid lines). A magnified view of the Al- $\mathrm{K}_{\alpha}$ peak is shown in Fig. 4c. A decrease in $\mathrm{Al}$ intensity of $\sim 5 \times 10^{-3}$ or $12 \%$ with respect to the grain interior is observed at the GB. The standard deviation of the noise level of the difference spectrum around the $\mathrm{Al}-\mathrm{K}_{\alpha}$ peak is $\sim 1.3 \times 10^{-3}$, which is nearly $\times 5$ smaller than the peak maximum. This analysis suggests that $\mathrm{Al}$ is depleted at the $\mathrm{GB}$, although first-principles calculations predicted a tendency for $\mathrm{Al}$ to segregate to substitutional sites at an idealized $\Sigma 5\left(\begin{array}{lll}3 & 1 & 0\end{array}\right) \mathrm{GB}$ in bcc-Fe. Any sign of enrichment of other impurity elements were not revealed in the STEM-EDS data. Since measuring light elements is difficult in STEM-EDS, APT was used in addition to get a precise insight of the GB chemistry.

Grain boundary composition. To obtain insights in the local distribution of $\mathrm{Al}$ and other impurity elements such as $\mathrm{C}$ and $\mathrm{B}$ at the GB with highest possible elemental sensitivity, we performed correlative STEM-APT investigations of the GB. Figure 5a presents a low magnification HAADF-STEM image of the needle shaped APT specimen containing the $\Sigma 5 \mathrm{~GB}$ at a distance of $\sim 100 \mathrm{~nm}$ from the apex. From the width of the needle-shaped specimen, the thickness of the tip at the boundary region can be estimated to $\sim 70 \mathrm{~nm}$. The viewing direction is along the $\left[\begin{array}{lll}0 & 0 & 1\end{array}\right]$ tilt axis. Figure $5 \mathrm{~b}$ shows the distribution of $\mathrm{Al}, \mathrm{C}$ and $\mathrm{B}$ atoms. In contrast to $\mathrm{C}$ and $\mathrm{B}$ enrichment at the $\mathrm{GB}$, a depletion of $\mathrm{Al}$ is apparent. A linear 1D composition profile extracted from a cylindrical region with $20 \mathrm{~nm}$ in diameter (see Fig. 5b) positioned perpendicular to the GB is shown in Fig. 5c. The Al concentration decreases from $\approx 3.8$ at $\%$ in the bulk (which is in agreement to our wet chemical analysis) to $\approx 2.4$ at $\%$ at the GB. In contrast, the C and B concentration increase to 2.4 at.\% and 1.8 at.\%, respectively. A more profound analysis provides information about the Gibbs interfacial excess (IE) $\Gamma_{i}$ of solute $i$, which describes the number of atoms per unit area at interfaces ${ }^{9}$. The measurements show that $\Gamma_{B} \approx 2.4$ atoms $\mathrm{nm}^{-2}$ and $\Gamma_{C} \approx 3.1$ atoms $\mathrm{nm}^{-2}$, where $\approx 8$ atoms $\mathrm{nm}^{-2}$ correspond to one monolayer. It should be noted that the IE value could increase when the diameter of the cylinder was reduced and placed across a solute enriched region within the $\mathrm{GB}$, indicating that the elemental distribution is inhomogeneous. For $\mathrm{Al}$ a negative IE value of $\Gamma_{A l} \approx-3.1$ atoms $\mathrm{nm}^{-2}$ was obtained.

For better understanding of $\mathrm{C}$ and $\mathrm{B}$ distribution, a second APT specimen was extracted from a location $\sim 6 \mathrm{~mm}$ apart from the previous STEM-APT observations. Figure 6a shows the reconstructed $3 D$ volume of the needle shaped specimen, where this time the GB plane was chosen to be perpendicular to the evaporation direction ([ $\left[\begin{array}{lll}0 & 0 & 1\end{array}\right]$ points towards the evaporation direction). Again, a clear enrichment of C (brown) and B (blue) at the GB were observed, but no segregation of Al. Rotation of the reconstruction by $90^{\circ}$ (see Fig. 6b) is showing the solute distribution along the GB plane, i.e. viewing along the [3 10$]$ direction. The distribution of $B$ and $C$ within the GB plane seems 

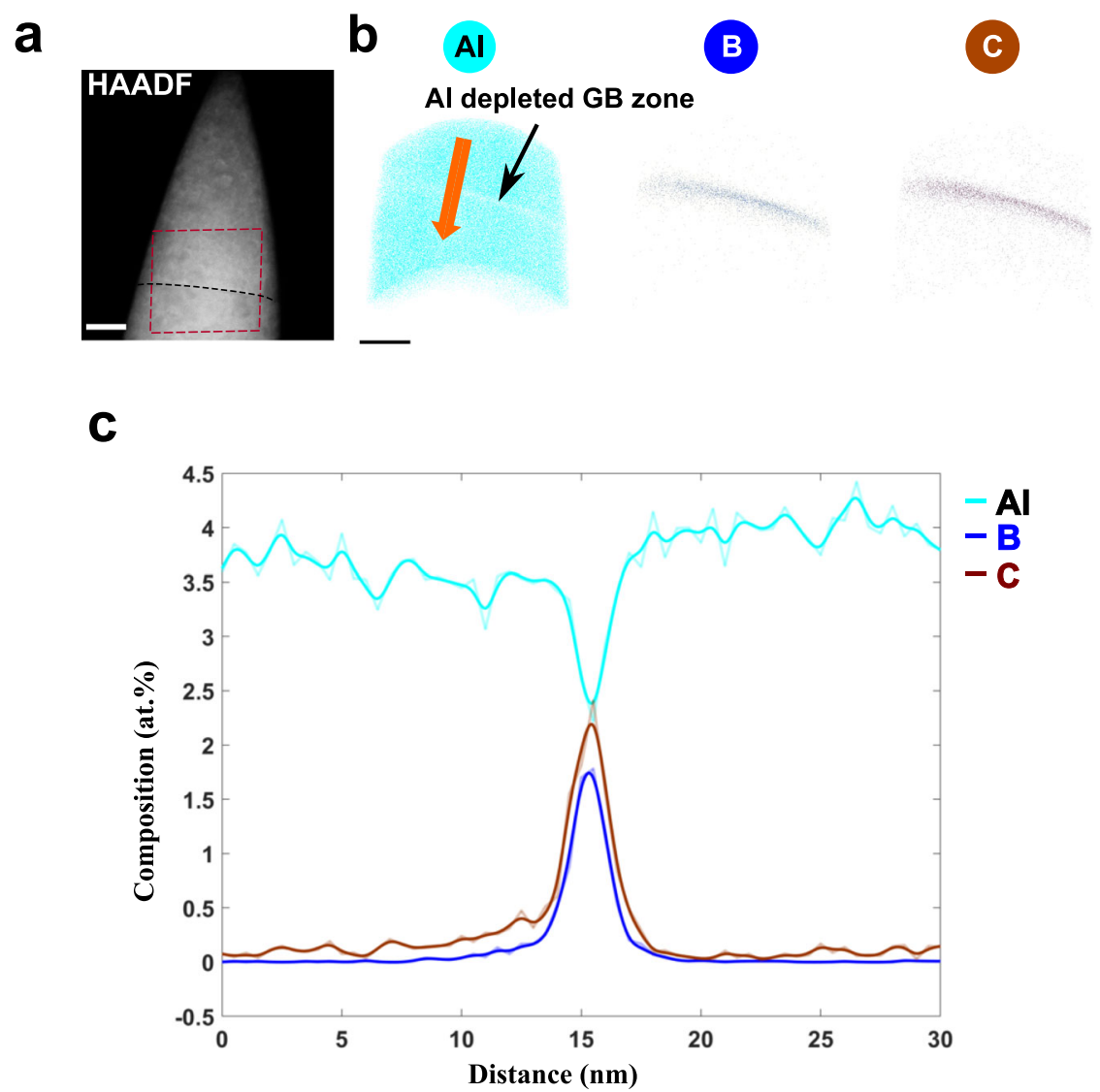

Fig. 5 Correlative STEM-APT study of the $\mathbf{\Sigma} \mathbf{5}$ GB. a HAADF-STEM image of the needle shaped APT specimen with both grains in [0 01 ] orientation. The $\mathrm{GB}$ is labeled with a black dashed line. $\mathbf{b}$ The atom maps of $\mathrm{Al}, \mathrm{B}$ and $\mathrm{C}$ reconstructed from the volume outlined by a red rectangle in $\mathrm{a}$ ). At the $\mathrm{GB}$, a depletion of $\mathrm{Al}$ and enrichment of $\mathrm{B}$ and $\mathrm{C}$ is observed. $\mathbf{c}$ The corresponding composition profiles extracted from a cylindrical region marked by an orange arrow in (b) across the GB shows a decrease of $A l$ and increase of B and C concentration at the GB. Scale bar in (a) is $20 \mathrm{~nm}$ and in (b) $10 \mathrm{~nm}$.

to be different with $\mathrm{C}$ showing a rather periodic segregation pattern with $C$ rich segments every $25 \mathrm{~nm}$. The composition profile along the GB (green arrow in a)) is shown in Fig. $6 c$ confirming a difference in the segregation behavior between $\mathrm{C}$ and B. A modulating distribution of $\mathrm{C}$ with an amplitude of $\sim 1$ at $\%$ and a periodicity of $25 \mathrm{~nm}$ was obtained. On the other hand, the average content of $B$ is $\sim 1$ at $\%$ with no significant modulation.

Although $\mathrm{Al}$ exhibits a high solubility of $4 \%$ at room temperature in bcc-Fe $\mathrm{F}^{53}$ a clear depletion at the GB was observed, which is in contradiction to previous computational ${ }^{32,33,54}$ and experimental ${ }^{55,56}$ studies. The complex co-segregation of $\mathrm{C}$ and $\mathrm{B}$ revealed by APT in this study indicates that those interstitial elements might strongly affect the segregation behavior of $\mathrm{Al}$.

Computational results. We performed DFT calculations to explore the interaction of solutes at the $\Sigma 5(310) \mathrm{GB}$ and their effect on segregation energies. A particular focus was laid on the interaction of $\mathrm{C}$ and $\mathrm{B}$ with $\mathrm{Al}$ to elucidate the observed $\mathrm{Al}$ depletion. Furthermore, the segregation kinetics are investigated to obtain insights into the complex co-segregation during bicrystal fabrication.

The pristine $\Sigma 5\left(\begin{array}{lll}3 & 1 & 0\end{array}\right)\left[\begin{array}{lll}0 & 0 & 1\end{array}\right]$ GB was modeled with 78 atoms in the unit cell and the ground state structure was determined using the $\gamma$-surface approach ${ }^{57}$ at $0 \mathrm{~K}$. The present DFT calculations do not include temperature effects from thermal excitations, which have been shown to be minor for the considered temperatures ${ }^{58,59}$. However, the effective segregation entropy that arises from averaging a multi-site GB excess to obtain a singular effective segregation energy ${ }^{36,60,61}$ is included in the analysis. The resulting structure viewed along the [ $\left[\begin{array}{lll}0 & 0 & 1\end{array}\right]$ tilt axis is shown in Fig. 7a. In the first step we have calculated the segregation energies of $\mathrm{Al}, \mathrm{C}$, and $\mathrm{B}$ at the GB using the equation

$$
E_{s e g, i}=\left(E_{g b, i}^{x}-E_{g b}\right)-\left(E_{b u l k}^{x}-E_{b u l k}\right)
$$

where $E_{g b}$ is the total energy of the pristine GB and $E_{g b, i}^{x}$ denotes the total energy of the GB cell with the solute $x$ at GB site $i$. The site $i$ is varied over positions shown in Fig. 7a. The reference of the solute in the bulk is given by the energy difference of $E_{b u l k}$, i.e. a cubic bulk cell that contains $128 \mathrm{Fe}$ atoms, and $E_{b u l k}^{x}$, which corresponds to the total energy of the bulk cell with solute $x$ at its preferred position. The position in the bulk is substitutional for $\mathrm{Al}$, while for $\mathrm{C}$ and $\mathrm{B}$ it is the octahedral interstitial site ${ }^{62}$. Because the atomic volume of $B$ is in between typical substitutional and interstitial solutes, we consider $B$ as both interstitial and substitutional at the GB. For the computation of the segregation energy, the number of atoms needs to be kept constant, which is the case in Eq. (1) if the site type is the same in the bulk and at the GB, i.e. either interstitial or substitutional. For placing $B$ in a substitutional site at the GB and in an interstitial site in the bulk, the segregation energy needs to be compensated by the energy of a single Fe atom in the bulk $E_{F e}=E_{b u l k} / 128$ :

$$
E_{\text {seg }, i}=\left(E_{g b, i}^{x}-E_{g b}\right)-\left(E_{b u l k}^{x}-E_{b u l k}\right)+E_{F e}
$$

A negative segregation energy of a solute indicates that the solute favors segregation to the GB.

We computed segregation energies at a coverage of 0.5 monolayers (ML) for Al to sites S1-S3, for C to sites I1-I4 and 
a

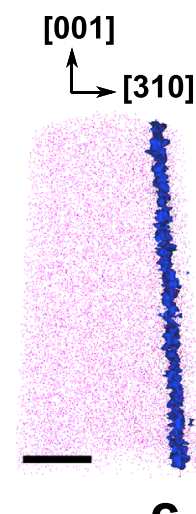

b

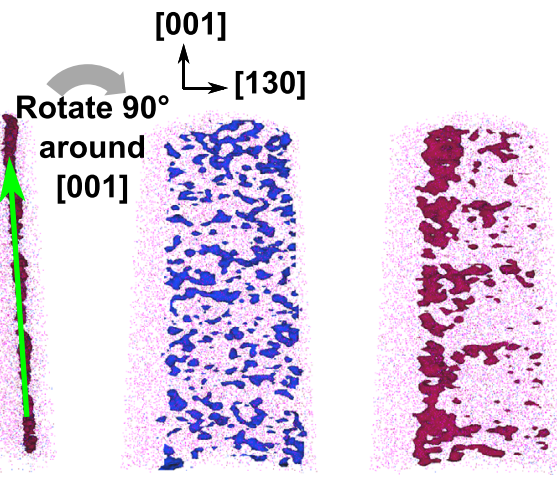

C

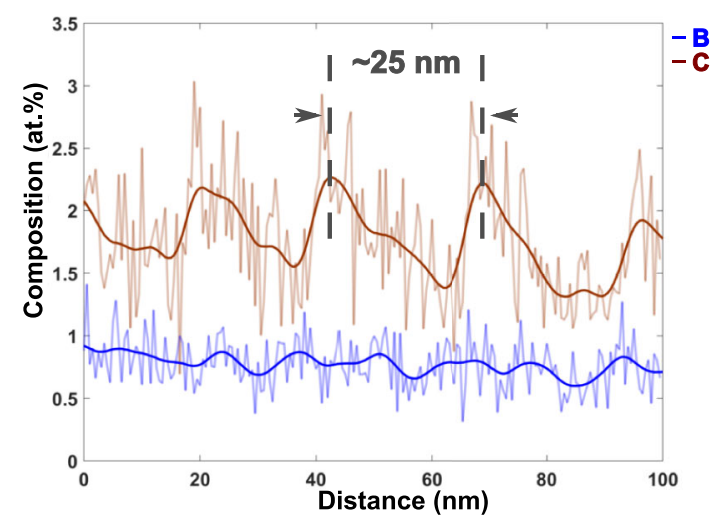

Fig. 6 Distribution of $\mathbf{C}$ and $\mathbf{B}$ along the $\mathbf{\Sigma} 5(\mathbf{3 1 0}) \mathbf{G B}$. a 3D APT reconstruction showing atom maps of $\mathrm{Fe}, \mathrm{Al}, \mathrm{B}$, and $\mathrm{C}$ as well as isoconcentration surfaces of 1 at $\%$ for $C$ (brown) and B (blue) viewed perpendicular to the GB plane. $\mathbf{b}$ The same APT reconstruction rotated by $90^{\circ}$ around [0 01 ] such that the viewing direction is along the (310) GB plane. c Corresponding composition profile of the GB extracted in a cylinder with $5 \mathrm{~nm}$ diameter along the green arrow in (a). While the $\mathrm{B}$ concentration is nearly constant at $\sim 1$ at $\%$ along the boundary, the $\mathrm{C}$ concentration varies periodically. The average distance between the regions with a peak C concentration of 2.3 at\% is determined to $25 \mathrm{~nm}$. Scale bar in (a) is $20 \mathrm{~nm}$.

a

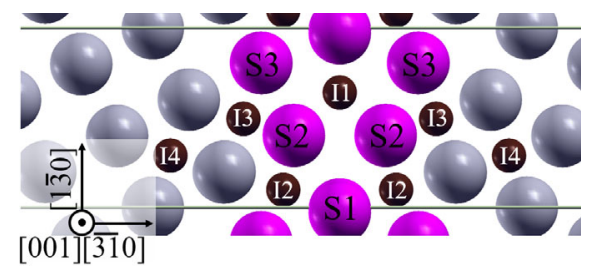

b

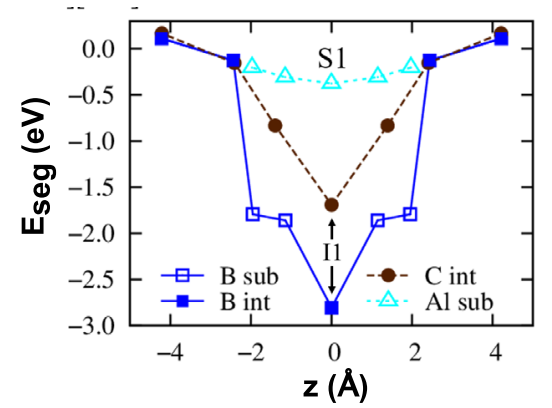

Fig. 7 Solute segregation energies at the GB. a shows the GB (highlighted by the purple atoms) with the interstitial sites (11-14), where (c and $\mathbf{b}$ ) were placed, and substitutional sites (S1-S3). b contains a plot of segregation energies of Al to substitutional sites (empty symbols), c to interstitial sites (solid symbols), and B to both substitutional and interstitial sites. for $\mathrm{B}$ to all labeled sites in Fig. 7a. The resulting segregation energies for $\mathrm{Al}, \mathrm{B}$, and $\mathrm{C}$ are given in Fig. 7b. By far the strongest segregation tendency is observed for $\mathrm{B}$ with a segregation energy of $-2.8 \mathrm{eV}$ to the interstitial site $\mathrm{I} 1$ at the GB center. The second strongest segregation tendency is found for the substitutional site $\mathrm{S} 2$ next to the $\mathrm{GB}$ center with $-1.86 \mathrm{eV}$. For $\mathrm{C}$ and $\mathrm{Al}$ the strongest segregation tendency is observed to the GB center with $-1.7 \mathrm{eV}$ in the case of $\mathrm{C}$ for position $\mathrm{I} 1$ and only $-0.38 \mathrm{eV}$ for $\mathrm{Al}$ for position $\mathrm{S} 1$, respectively. The segregation tendency decreases with increasing distance from the GB. When placing $\mathrm{B}$ at the segregation site $\mathrm{I} 2$, the $\mathrm{B}$ atom moved to site I1.

The segregation tendency of B to substitutional sites S2 and S3 at the GB is substantial, but it also introduces a strong displacement of the surrounding Fe atoms and hence a distortion of the kite structure as seen in Fig. 8. Especially for B at site S2, the B induced distortion is significant. This can be quantified by the mean squared displacement (MSD) of the atoms in the vicinity of $\mathrm{B}$ in a radius of $5 \AA$. For B at site S1, S2, and S3 the MSD is $0.14,0.40$, and $0.59 \AA$, respectively. The corresponding simulated HAADF-STEM images using the Prismatic software ${ }^{63,64}$ are shown in the lower panels of Fig. 8. Here, the simulated HAADF contrast predominately shows the $\mathrm{Fe}$ atomic columns and the $\mathrm{B}$ and/or $\mathrm{C}$ atoms are only indirectly visible. This can be seen in the slight decrease in intensity of the atomic columns in the kite tips when B is segregating to the substitutional site $\mathrm{S} 1$ (see Fig. $8 \mathrm{a}$ ). When $\mathrm{B}$ is segregating to position S2 (Fig. 8b), the neighboring Fe atoms are displaced causing a smearing of the HAADF contrast and the 

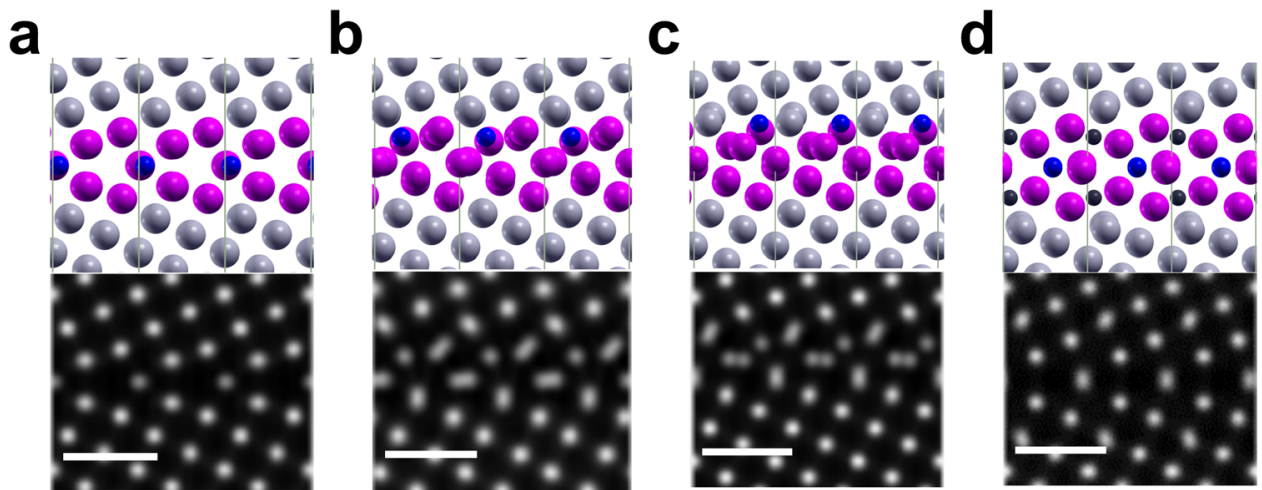

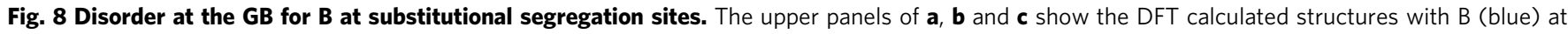
substitutional sites 1, 2, 3. The lower panels are the corresponding HAADF-STEM simulated structures. $\mathbf{d}$ shows the GB segregated with two B (in one column) and two C (brown) atoms from Fig. 9 f (see below). The scale bar is 500 pm.

individual columns are no longer resolvable. An even stronger displacement of the Fe atoms is introduced when B is located at site S3, as seen in the top panel of Fig. 8c, and here Fe dumbbells are observed at the kite tips in the simulated HAADF-STEM image (lower panel of Fig. 8c). Even placing $\mathrm{B}$ as well as $\mathrm{C}$ in the intersitial positions I1 and I2 (Fig. 8d) can have a slight distortion of the kite tips, where the Fe atoms are shifted perpendicular to the boundary plane.

So far, the computed segregation energies are obtained for individual solutes, but the interaction of solutes can have significant effects on the segregation tendencies when the binding energies are in the range of the segregation energies ${ }^{65,66}$. In the following section, we explore the co-segregation behavior of $\mathrm{Al}$ with both $\mathrm{C}$ and $\mathrm{B}$ as a possible explanation for the observed depletion of $\mathrm{Al}$ from the GB. To that end, we have computed the binding energies of $\mathrm{Al}$ at its preferred site $\mathrm{S} 1$ in the $\mathrm{GB}$ center to $\mathrm{B}$ and $\mathrm{C}$ at vicinal interstitial segregation sites (I1-I4). The binding energy between $\mathrm{Al}$ and a solute $x$ is defined as

$$
E_{\text {bind }}^{A l-x}=\left(E_{A l}+E_{x}\right)-\left(E_{A l-x}+E\right)
$$

where $E_{A l-x}$ denotes the total energy of the GB cell with $\mathrm{Al}$ and the solute $x$ present. $E_{A l}$ and $E_{x}$ are the energies of the same cell with either Al or solute $x$ placed at their lowest energy segregation sites, respectively. $E$ is the energy of the simulation cell not containing any solutes. For attractive interactions, the binding energy is positive, while repulsive interactions are associated with negative binding energies.

Figure $9 \mathrm{a}-\mathrm{d}$ shows exemplary the calculated GB structure with B and $\mathrm{C}$ atoms placed in different intersitial (I1-I4) and substitutional (S1-S3) sites. The binding energies of $\mathrm{Al}$ in its preferred position and either $\mathrm{B}$ or $\mathrm{C}$ are shown in Fig. 9e. For $\mathrm{Al}$ and $\mathrm{B}$ in their lowest energy sites, S1 and I1, respectively, the binding energy is determined to $-0.40 \mathrm{eV}$, which is repulsive. If $\mathrm{B}$ is considered in one of the substitutional sites, the binding energy varies between $-0.42 \mathrm{eV}$ ( $\mathrm{Al}$ in $\mathrm{S} 1$ and $\mathrm{B}$ in $\mathrm{S} 2$ ) and $-0.24 \mathrm{eV}$ ( $\mathrm{Al}$ in S1 and $\mathrm{B}$ in $\mathrm{S} 3$ ). For $\mathrm{C}$, multiple repulsive constellations are found with binding energies in the range of -0.70 to $-0.40 \mathrm{eV}$ for small distances of $<2.5 \AA$ between $\mathrm{Al}$ and $\mathrm{C}$. For the constellation of $\mathrm{Al}$ at site $\mathrm{S} 1$ and $\mathrm{C}$ at site $\mathrm{I} 2$, with a distance of $\sim 3.3 \AA$, a slightly positive binding energy is obtained. However, this is not the site where $\mathrm{C}$ exhibits the strongest segregation tendency. For both $\mathrm{C}$ and $\mathrm{B}$ interaction with $\mathrm{Al}$, the magnitudes of the binding energies are mostly larger than the segregation tendency of $\mathrm{Al}$ to the GB. The strong repulsive interactions of $\mathrm{Al}$ with either $\mathrm{B}$ or $\mathrm{C}$ imply that cosegregation of these elements is highly unlikely. This suggests that $\mathrm{Al}$ is repelled from the $\mathrm{GB}$, since both $\mathrm{C}$ and $\mathrm{B}$ exhibit a much stronger segregation tendency in comparison to $\mathrm{Al}$.
Another effect that comes into play with solute interactions is the coverage dependence, which refers to changes in the segregation energy with increasing coverage at the GB. This may be computed using Eqs. (1) and (2), where $E_{g b, i}^{x}$ denotes the total energy of the structure with $\mathrm{N}+1$ solutes at the GB and $E_{g b}$ the structure with $\mathrm{N}$ solutes at the GB. For the lowest solute coverage we take $0.5 \mathrm{ML}$ which corresponds to the results shown in Fig. 7 and add atoms to preferential segregation sites. The resulting coverage dependent segregation energies are displayed in Fig. 9f. As B has by far the strongest segregation energy, the starting structure contains a B atom at the GB. Adding a second B atom leads only to a slightly lower segregation tendency for the interstitial positions $\left(2 \mathrm{~B}_{\mathrm{I}}\right)$, whereas in the substitutional case, the decrease is somewhat stronger $\left(\mathrm{B}_{\mathrm{S} 2}\right.$ to $\left.2 \mathrm{~B}_{\mathrm{S} 2}\right)$. Adding a third $\mathrm{B}$ atom, however, leads to a large decrease in segregation tendency to even positive values (the lowest energy configuration $2 \mathrm{~B}_{\mathrm{I} 1}-\mathrm{B}_{\mathrm{S} 3}$ is at $+0.22 \mathrm{eV}$ ). This means that additional $\mathrm{B}$ segregation above a coverage of 8 atoms $\mathrm{nm}^{-2}$ becomes unfavorable. When adding $\mathrm{C}$ to the GB to site $\mathrm{I} 2$ with already two $\mathrm{B}$ atoms present in position I1 gives a negative segregation energy of $-0.42 \mathrm{~nm}$. Even for an additional $\mathrm{C}$ atom in $\mathrm{I} 2$, a negative segregation energy of $-0.33 \mathrm{eV}$ is obtained. This indicates that although B shows strong segregation tendencies to different positions at the GB, at $1 \mathrm{ML}$ coverage or about 8 atoms $\mathrm{nm}^{-2}$ a maximum enrichment is reached due to solute interactions.

The enrichment of solutes at GBs in equilibrium can be described with a modified McLean isotherm, but under experimental conditions kinetic processes often influence GB segregation. Therefore, we compute the enrichment of solutes at the GB by considering segregation kinetics via our recently published model $^{54,67}$. Note that the slow cooling rate during bicrystal growth minimizes non-equilibrium segregation effects as described by Faulkner ${ }^{68}$ as possible origin for solute enrichment at grain boundaries, and therefore, these effects are not included in our modeling approach. As input parameters we use grains with a radius of $100 \mu \mathrm{m}$ that is further discretized into shells of $1 \mu \mathrm{m}$ thickness. Although the bi-crystals are in the range of $\mathrm{cm}$, the system size is sufficient because the diffusion length of all involved solutes is below $100 \mu \mathrm{m}$ in the chosen heat treatment. The GB thickness is taken as $0.8 \mathrm{~nm}$, which is the region where segregation energies are negative. The diffusion data ${ }^{69}$ is given in the Supplementary Table 1 and for the heat treatment we consider cooling down from $1800 \mathrm{~K}$ with the experimentally informed ${ }^{67}$ modified Newton's law of cooling. The cooling rate of $r=0.017 \mathrm{~s}^{0.5}$ is chosen such that room temperature is reached after $24 \mathrm{~h}$ (see Fig. 10a) similar to the fabrication of the bi-crystal specimen. The bulk concentration of the solutes was taken from 

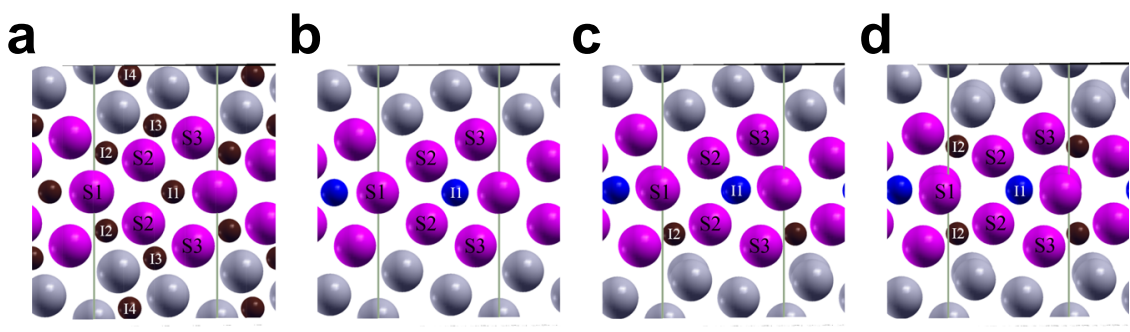

\section{$\mathbf{e}$}
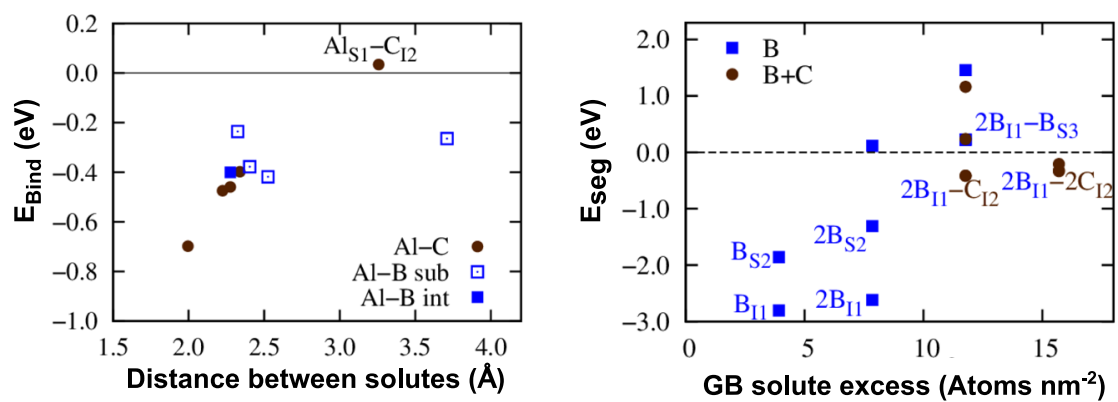

Fig. 9 Solute interactions at GB. a GB structure with labeled substitutional (S1-S3) and interstitial sites (I1-I4). b The GB structure corresponding to configuration $B_{11}$ and $2 B_{11}$ (the two $B$ atoms are oriented in $\left[\begin{array}{ll}0 & 0\end{array}\right]$ direction and the structure cannot be distinguished from configuration $B_{11}$ ). $\mathbf{c}$, $\mathbf{d}$ shows the configurations $2 B_{11} C_{12}$ and $2 B_{11} 2 C_{12}$. In $\mathbf{e}$, the binding energies of $A$ l with $B$ and with $C$ are shown as a function of the distance between the solutes. Panel $\mathbf{f}$ presents the co-segregation energies of $B$ and $C$ atoms to the $G B$, where important configurations are named.

the wet chemical analysis. The segregation energies necessary were taken from the coverage dependence shown in Fig. 9f, i.e. for $\mathrm{B}$ site $\mathrm{I} 1$ is considered and for $\mathrm{C}$ the site $\mathrm{I} 2$. We want to point out that it is not possible to include the computed solute-solute interactions with classical approaches like the Guttmann or Fowler isotherms ${ }^{68}$ as these take as input only a single value, whereas we find that each configuration has its own individual interaction energy. Rather Monte Carlo Simulations would be required to link to the DFT data. Such calculations are beyond the scope of this study

The resulting time and temperature dependent enrichment of solutes at the GB is shown in Fig. 10. Due to the strong repulsive interactions of $\mathrm{Al}$ with $\mathrm{B}$ and $\mathrm{C}$, we investigate $\mathrm{Al}$ segregating on its own in the center panel (Fig. 10b), while we consider mutual segregation for $\mathrm{B}$ and $\mathrm{C}$ in the bottom panel (Fig. 10c). The GB enriches with $\mathrm{Al}$ for temperatures above $800 \mathrm{~K}$, but for temperatures below, segregation is kinetically limited. At this stage, the excess concentration of $\mathrm{Al}$ is 22 atoms $\mathrm{nm}^{-2}$. Here it is important to note that for $\mathrm{Al}$ no coverage dependency was considered. For B, the GB enrichment takes place relatively fast at high temperatures because of the strong segregation tendencies and stops at a GB excess of 7 atoms $\mathrm{nm}^{-2}$ for temperatures below $1200 \mathrm{~K}$ due to kinetic reasons. This happens already for higher temperatures than for $\mathrm{Al}$ because the concentration in the bulk is much lower than for $\mathrm{Al}$ and for $\mathrm{GB}$ enrichment, $\mathrm{B}$ needs to diffuse from farther away from the GB. Contrary, $C$ is able to enrich until temperatures of only $390 \mathrm{~K}$ due to faster diffusion and higher content in the bulk than for $\mathrm{B}$ so that a final GB excess of 16 atoms $\mathrm{nm}^{-2}$ is reached. The fact that B enriches already at higher temperatures than $\mathrm{Al}$ reduces the possibility of $\mathrm{Al}$ segregation even more because $\mathrm{B}$ is already present at the GB in a temperature range where $\mathrm{Al}$ segregation takes place.

\section{Discussion}

In this study, the atomic structure, composition and cosegregation mechanisms of $\mathrm{Al}, \mathrm{C}$ and $\mathrm{B}$ at a $\Sigma 5\left(\begin{array}{lll}3 & 1 & 0\end{array}\right)\left[\begin{array}{lll}0 & 0 & 1\end{array}\right]$
bcc-Fe GB were investigated by correlating aberration corrected HAADF-STEM observations, APT measurements and firstprinciples DFT calculations. The observed atomic structure of the $\Sigma 5\left(\begin{array}{lll}3 & 1 & 0\end{array}\right)\left[\begin{array}{lll}0 & 0 & 1\end{array}\right]$ GB consists of kite-type structural units and agrees to investigations by Medlin et al. ${ }^{39}$. Globally, the GB appeared straight and close to the symmetric orientation, but atomic resolved observations indicated the presence of defects such as steps or disconnections. Further, in close vicintiy to a GB precipitate steps are incorporated into the GB to compensate the local curvature. Interestingly, this GB segment does not break up into $\left\{\begin{array}{lll}2 & 1 & 0\end{array}\right\}$ and $\left\{\begin{array}{lll}3 & 1 & 0\end{array}\right\}$ facets (as observed by Medlin et al. ${ }^{39}$ ), but retains the $\left(\begin{array}{ll}3 & 1\end{array}\right)$ ) kite-type structural units. The reason is that the inclination angle of only $7^{\circ}$ from the symmetric orientation seems not to be sufficient to promote faceting.

Our compositional analysis of the GB revealed a depletion of $\mathrm{Al}$ at the interface, where locally the $\mathrm{Al}$ concentration reduces by $\sim 1.5$ at $\%$ at the GB. This is in contradiction to first-principles calculations of Yasua et al. ${ }^{32}$ and Geng et al. ${ }^{33}$ as well as Scheiber et al. ${ }^{54}$, who observed the segregation of Al. However, it should be mentioned, that they investigated a pure $\mathrm{Fe}-\mathrm{Al}$ system. From our APT measurements, we were able to detect the segregation of B and C. Taking these elements into our calculations, we showed that both $\mathrm{C}$ and $\mathrm{B}$ have much stronger segregation tendencies than $\mathrm{Al}$ (Fig. 7), so that already simple site competition arguments suggest a depletion of $\mathrm{Al}$ due to competition with $\mathrm{B}$ for substitutional sites. Explicit calculation of solute interactions revealed strong repulsive interactions between $\mathrm{Al}-\mathrm{C}$ and $\mathrm{Al}-\mathrm{B}$. By considering the kinetics of segregation, it is clear that $\mathrm{B}$ is the first solute to segregate to the GB, which prevented $\mathrm{Al}$ enrichment and explained the depletion of Al. The composition evaluated with the kinetic model and first principles segregation energies is substantially higher than the composition observed with APT. This is attributed to co-segregation effects between $\mathrm{B}$ with itself, $\mathrm{C}$ with itself and B with $\mathrm{C}$. In fact, evaluation of these co-segregation effects showed that increased coverage of B decreases the segregation tendency at coverages above $1 \mathrm{ML}$. It is not possible to implement these interactions into the current kinetic model, still, 


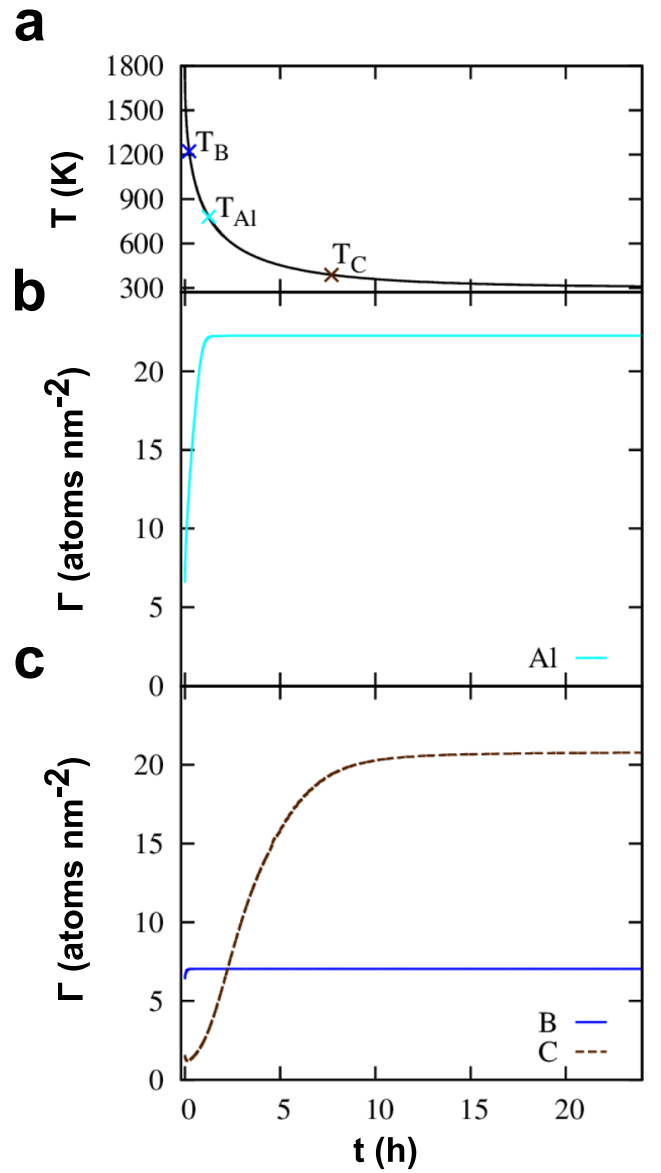

Fig. 10 Computed grain boundary segregation kinetics. a Timetemperature curve during cooling with the temperatures $T_{\text {sol }}$ marked at which segregation for the solutes is limited kinetically. $\mathbf{b}$ The resulting enrichment of $\mathrm{Al}$ atoms without interaction with other solutes. c Competing temperature dependent segregation of $C$ and $B$ while the bottom panel shows the enrichment for competing $B$ and $C$ segregation.

it can be inferred that co-segregation effects limit enrichment of $\mathrm{B}$ and $C$. The observed enrichment kinetics should hold true nonetheless, i.e., a first fast enrichment of B, followed by slower enrichment of $\mathrm{C}$ that continues on until lower temperatures. Even though co-segregation effects are known in general ${ }^{70}$, they were not observed in the case of bcc-Fe- $\mathrm{Al}(\mathrm{C}, \mathrm{B})$ and never explained in a comprehensive manner by combining atomistic simulations and experimental observations.

Another interesting finding is the observation of locally distorted kite structure. Its origin can be related to the presence of defects, which are aligned along the tilt axis, and to segregation of $B$ to substitutional sites. The assumption that GB defects alter the projected structural units is supported by observations of a periodic distribution of $\mathrm{C}$ in our APT analysis. It is known that $\mathrm{C}$ preferentially segregates to highly distorted regions such as GB dislocation cores ${ }^{71}$. These defects could also be responsible for the extra contrast observed in the open volume of the kites (indicated by yellow arrows in Fig. 2 b). Hyde et al. ${ }^{72}$ reported that by the formation of GB dislocation loops a metastable structure having extra $\mathrm{Fe}$ atoms in the kite center may appear. The distortion of the kites can also have a compositional origin. Our DFT simulations showed that different substitutional sites are also preferred positions for B segregation introducing strong displacements of $\mathrm{Fe}$ atoms from their equilibrium positions (Fig. 8). STEM image simulations of these structures-especially panel $b$ of Fig. 8-closely resemble our observations in the red dashed line in
Fig. $2 \mathrm{~b}$ even so the individual $\mathrm{B}$ atoms can not be localized. Furthermore, the formation of $\mathrm{Fe}$ dumbbells in the simulated images induced by B segregation could explain the weak contrast in the kite center observed in the experiments (see yellow arrows in the inset of Fig. 2b. The weaker contrast originates that we do not have a full column of Fe atoms shifted, but partly. Therefore it is highly possible that defects in combination to the B segregation is responsible for the distorted structure of the GB.

Our results demonstrate that co-segregation effects in the presence of impurities leads to unexpected GB segregation effects and possible GB structural transformations. Considering the interaction of non-metallic elements, here $\mathrm{B}$ and $\mathrm{C}$, with substitutional alloying additions, in our case $\mathrm{Al}$, is indispensable to fully capture the entire segregation processes. We discovered that the presence of $B$ and $C$ impurities segregated to the $G B$ is repelling $\mathrm{Al}$ from the $\mathrm{GB}$ by strong repulsive interactions. Furthermore, the competing mobilities of solutes critically influences the evolution of GB composition. We ultimately show that the presence of non-metallic impurity elements needs to be considered when studying GB segregation effects. Additionally, we expect similar trends in segregation of $B$ and $C$ even to general GBs in polycrystalline materials, which typically adopt complex structural arrangements and contain a high number of defects (such as disconnections and facets). This is supported by observations made by Janovec et al. ${ }^{73}$ who compared segregation tendencies in Fe-Si-P polycrystal as well as in a bicrystal of the same composition. They even found a stronger segregation of $\mathrm{P}$ in general GBs. Moreover, Yu et al. ${ }^{74}$ observed periodic segregation patterns even at general GBs in the $\mathrm{Ni}-\mathrm{Bi}$ system and identified the GB plane normal as the driving force for the formation of such periodic enrichment of $\mathrm{Bi}$ in Ni polycrystals. Therefore, our work also paves the way for tailoring GB composition by tuning impurity additions.

\section{Methods}

Experimental. For the experiments, bicrystals of an Fe-Al alloy having a $\Sigma 5\left[\begin{array}{lll}0 & 0 & 1\end{array}\right]$ GB were grown using an in-house modified Bridgman technique. In this method two single crystal seeds are mounted on a Fe-Al polycrystal. The seeds were aligned by their common $\left[\begin{array}{lll}0 & 0 & 1\end{array}\right]$ axis and misoriented by an angle of $\Theta \approx 38^{\circ}$ along their common [ $\left[\begin{array}{lll}0 & 0 & 1\end{array}\right]$ axis. The polycrystal is melted at a temperature of $1600^{\circ}$ under high vacuum $\left(p \approx 10^{-6} \mathrm{mbar}\right)$ in a vertical Bridgman furnace by slowly moving the heating coils upwards with a speed of $2 \mathrm{~mm} / \mathrm{h}$. This leads to a slow solidification of the melting zone and the formation of a bicrystal containing a single GB. After the growth process the temperature of the oven is decreased such that after $\approx 24 \mathrm{~h}$ room temperature is reached. The final cylindrical bicrystal has a diameter of $20 \mathrm{~mm}$ and contains the GB in its center over a total length of $100 \mathrm{~mm}$. Supplementary Table 2 shows results of the wet chemical analysis from a $2 \mathrm{~mm}$ slice cut using spark erosion. Besides the 4 at $\mathrm{Al}$ to stabilize the bcc phase ${ }^{53}$, the wet chemical analysis of the bulk sample revealed a content of 0.001 at\% B and 0.05 at\% C.

A second $5 \mathrm{~mm}$ slice was cut from the initial part of the bicrystal (indicated by pruple lines in Supplementary Fig. 1a). The $\left(\begin{array}{lll}0 & 0 & 1\end{array}\right)$ surface of the slice was mechanically polished to obtain a mirror-like area. To visualize the GB, the sample was etched with a solution of nitric acid: ethanol $=1: 9$ (volume fraction) for $5 \mathrm{~s}$. The GB showed globally a flat and straight character (Supplementary Fig. 1b). Information about the GB type were acquired form EBSD analysis (Supplementary Fig. 1b, c), which was carried out by means of a ThermoFisher Scios 2 dual beam FIB/SEM equipped with an EDAX Velocity EBSD camera.

From the characterized area, several TEM specimens were prepared by FIB. HAADF-STEM images were conducted using a $\mathrm{C}_{s}$ probe-corrected FEI Titan Themis $60-300$ operated at $300 \mathrm{kV}$ for imaging and $120 \mathrm{kV}$ for STEM-EDX. Using a semiconvergence angle of $17 \mathrm{mrad}$ and a camera length of $100 \mathrm{~mm}$ resulted in a semicollection angle range of 73 to $352 \mathrm{mrad}$. The probe current was set to $80 \mathrm{pA}$ to prevent beam damages. High resolution images were acquired in a series of at least 30 frames each recorded with a dwell time of $5 \mu \mathrm{s}$. These frames were stacked by means of cross correlation using the FEI Velox 2.8 software. STEM simulation were performed by the Prismatic Software package ${ }^{63}$. Finally, APT measurements were conducted in a CAMECA LEAP $5000 \mathrm{XR}$ operating in laser-pulsed mode. The pulse rate was $200 \mathrm{kHz}$ with a energy of $30 \mathrm{pJ}$ and the temperature of the specimen was $40 \mathrm{~K}$

Computational details. The first principles density functional theory calculations in this study were performed using the Vienna Ab-initio Simulation Package 
(VASP), which employ projector augmented wave-functions ${ }^{75-78}$. We have chosen the PBE exchange-correlation functional ${ }^{79,80}$ as it has been shown that it is suited best to describe $\mathrm{Fe}^{81}$. The k-point density for all involved simulation cells was set as close as possible to $40 \mathrm{k}$-points/ $\AA$ and the energy cutoff to $400 \mathrm{eV}$. As a result, the lattice parameter of $\mathrm{Fe}$ was determined as $2.8386 \AA$, which is in accordance with DFT literature ${ }^{57,82}$. The simulation cells for GBs are build up of two slabs that are misoriented to each other so that they form a GB where they join. On the other side, the slabs are separated by a vacuum layer of at least $8 \AA$ that proved to be large enough to prevent interactions of the two surfaces. With this setup, the simulation cell is constructed along the lattice vectors $\left[\begin{array}{lll}0 & 0 & 1\end{array}\right] \times\left[\begin{array}{lll}1 & -3 & 1\end{array}\right] \times\left[\begin{array}{ll}3 & 1\end{array}\right]$ with $5.677 \times 4.708 \times 44.883 \AA^{3}$ and contains $78 \mathrm{Fe}$ atoms. For ionic relaxations a convergence criterion of $0.01 \mathrm{eV} / \AA$ is employed.

\section{Data availability}

The data that support the findings of this study are available from the corresponding author upon reasonable request.

\section{Code availability}

The code that support the findings of this study are available from the corresponding author upon reasonable request.

Received: 21 December 2020; Accepted: 13 September 2021; Published online: 14 October 2021

\section{References}

1. Papazian, J. M. \& Besherb, D. N. Grain boundary segregation of carbon in iron. Metall. Trans. 2, 497-503 (1971).

2. Suzuki, S., Obata, M., Abiko, K. \& Kimura, H. Effect of carbon on the grain boundary segregation of phosphorus in $\alpha$-iron. Scr. Metall. 17, 1325-1328 (1983).

3. Hänsel, H. \& Grabke, H. J. Grain boundary segregation of phosphorus and carbon in ferritic iron. Scr. Metall. 20, 1641-1644 (1986).

4. Lejček, P. \& Hofmann, S. Temperature dependence of the phosphorus segregation at the twin boundary in an $\mathrm{Fe}-4$ at\% Si alloy. Surf. Interface Anal. 16, 546-551 (1990).

5. Lejček, P. \& Hofmann, S. Segregation enthalpies of phosphorus, carbon and silicon at $\{013\}$ and $\{012\}$ symmetrical tilt grain boundaries in an Fe-3.5 at $\% \mathrm{Si}$ alloy. Acta Metall. Mater. 39, 2469-2476 (1991).

6. Lejček, P. Characterization of grain boundary segregation in an Fe-Si alloy. Anal. Chim. Acta 297, 165-178 (1994).

7. Fraczkiewicz, A., Gay, A.-S. \& Biscondi, M. On the boron effect in FeAl (B2) intermetallic alloys. Mater. Sci. Eng. A 258, 108-114 (1998).

8. Fraczkiewicz, A. Influence of boron on the mechanical properties of B2Ordered FeAl Alloys. Mater. Trans. JIM 41, 166-169 (2000).

9. Krakauer, B. \& Seidman, D. N. Atomic Scale Observations of Solute-Atom Segregation at Grain Boundaries in an Iron (Silicon) Alloy. Mater. Sci. Forum 126-128, 161-164 (1993).

10. Maruyama, N., Smith, G. D. W. \& Cerezo, A. Interaction of the solute niobium or molybdenum with grain boundaries in $\alpha$-Iron. Mater. Sci. Eng. A 353, 126-132 (2003).

11. Seah, M. P. Grain boundary segregation. J. Phys. F: Met. Phys. 10, 1043-1064 (1980).

12. Xing, W., Kalidindi, A. R., Amram, D. \& Schuh, C. A. Solute interaction effects on grain boundary segregation in ternary alloys. Acta Mater. 161, 285-294 (2018).

13. Guttmann, M. Equilibrium segregation in a ternary solution: a model for temper embrittlement. Surf. Sci. 53, 213-227 (1975).

14. Ohtsuka, S., Shinoda, Y., Akatsu, T. \& Wakai, F. Effect of oxygen segregation at grain boundaries on deformation of B, C-doped silicon carbides at elevated temperatures. J. Am. Ceram. Soc. 88, 1558-1563 (2005).

15. Wang, Z. et al. Atom-resolved imaging of ordered defect superstructures at individual grain boundaries. Nature 479, 380-383 (2011).

16. Grabke, H. J. Surface and grain boundary segregation on and in iron. Steel Res. 57, 178-185 (1986).

17. Grabke, H. J., Paulitschke, W., Tauber, G. \& Viefhaus, H. Equilibrium surface segregation of dissolved nonmetal atoms on iron(100) faces. Surf. Sci. 63, 377-389 (1977).

18. Tauber, G. \& Grabke, H. J. Grain boundary segregation of sulfur, nitrogen, and carbon in $\alpha$-iron. Ber. Bunsenges. Phys. Chem. 82, 298-302 (1978).

19. Pichard, C., Rieu, J. \& Goux, C. The influence of oxygen and sulfur on the intergranular brittleness of iron. Metall. Trans. A 7, 1811-1815 (1976).

20. Liu, C. M., Nagoya, T., Abiko, K. \& Kimura, H. Effect of boron on the grain boundary segregation of phosphorus and intergranular fracture in high-purity Fe-0.2 Pct P-B alloys. Metall. Trans. A 23, 263-269 (1992).
21. Liu, C. T., White, C. L. \& Horton, J. A. Effect of boron on grain-boundaries in Ni3Al†. Acta Metall. 33, 213-229 (1985).

22. Painter, G. S. \& Averill, F. W. Effects of segregation on grain-boundary cohesion: a density-functional cluster model of boron and sulfur in nickel. Phys. Rev. Lett. 58, 234-237 (1987)

23. Wu, R., Freeman, A. J. \& Olson, G. B. First principles determination of the effects of phosphorus and boron on iron grain boundary cohesion. Science 265, 376-380 (1994).

24. Yamaguchi, M. First-principles study on the grain boundary embrittlement of metals by solute segregation: Part I. Iron (Fe)-solute (B, C, P, and S) systems. Met. Mat. Trans. A 42, 319-329 (2011).

25. Braithwaite, J. S. \& Rez, P. Grain boundary impurities in iron. Acta Mater. 53, 2715-2726 (2005).

26. Chen, Z.-Z. \& Wang, C.-Y. First-principles study on the effects of cosegregation of $\mathrm{Ti}, \mathrm{B}$ and $\mathrm{O}$ on the cohesion of the $\alpha$-Fe grain boundary. $J$. Phys.: Condens. Matter 17, 6645-6652 (2005).

27. Lejček, P. \& Fraczkiewicz, A. Boron segregation in intermetallics: On the possible origins of a low-level intergranular segregation. Intermetallics 11, 1053-1063 (2003).

28. Wu, R., Freeman, A. J. \& Olson, G. B. Effects of carbon on Fe-Grain-Boundary cohesion: First-Principles determination. Phys. Rev. B 53, 7504-7509 (1996).

29. Wang, J., Janisch, R., Madsen, G. K. H. \& Drautz, R. First-principles study of carbon segregation in bcc iron symmetrical tilt grain boundaries. Acta Mater. 115, 259-268 (2016).

30. Wachowicz, E. \& Kiejna, A. Effect of impurities on grain boundary cohesion in bcc iron. Comput. Mater. Sci. 43, 736-743 (2008).

31. Rana, R., Liu, C. \& Ray, R. K. Low-density low-carbon Fe-Al ferritic steels. Scr. Mater. 68, 354-359 (2013).

32. Yuasa, M. \& Mabuchi, M. First-principles study in Fe grain boundary with $\mathrm{Al}$ segregation: Variation in electronic structures with straining. Philos. Mag. 93, 635-647 (2013)

33. Geng, W. T., Freeman, A. J. \& Olson, G. B. Influence of alloying additions on grain boundary cohesion of transition metals: First-Principles determination and its phenomenological extension. Phys. Rev. B 63, 165415 (2001).

34. Rice, J. R. \& Wang, J.-S. Embrittlement of interfaces by solute segregation. Mater. Sci. Eng. A 107, 23-40 (1989).

35. Rellick, J. \& Mcmahon, C. Elimination of Oxygen-Induced Intergranular Brittleness in Iron by Addition of Scavengers. Metall. Trans. 1, 929-\& (1970).

36. Lejček, P. Grain Boundary Segregation in Metals. No. 136 in Springer Series in Materials Science (Springer-Verlag, Berlin, 2010).

37. Raabe, D. et al. Grain boundary segregation engineering in metallic alloys: A pathway to the design of interfaces. Curr. Opin. Solid State Mater. Sci. 18, 253-261 (2014).

38. Da Rosa, G., Maugis, P., Drillet, J., Hebert, V. \& Hoummada, K. Cosegregation of boron and carbon atoms at dislocations in steel. J. Alloy. Compd. 724, 1143-1148 (2017).

39. Medlin, D., Hattar, K., Zimmerman, J., Abdeljawad, F. \& Foiles, S. Defect character at grain boundary facet junctions: Analysis of an asymmetric $\Sigma=5$ grain boundary in Fe. Acta Mater. 124, 383-396 (2017).

40. Lejček, P. \& Adimek, J. Anisotropy of grain boundary segregation in $\Sigma=5$ bicrystals of $\alpha$-Iron. Surf. Sci. 264, 449-454 (1992).

41. Larson, D., Gault, B., Geiser, B., De Geuser, F. \& Vurpillot, F. Atom probe tomography spatial reconstruction: Status and directions. Curr. Opin. Solid State Mater. Sci. 17, 236-247 (2013).

42. Ashton, M., Mishra, A., Neugebauer, J. \& Freysoldt, C. Ab initio description of bond breaking in large electric fields. Phys. Rev. Lett. 124, 176801 (2020).

43. De Geuser, F. \& Gault, B. Metrology of small particles and solute clusters by atom probe tomography. Acta Mater. 188, 406-415 (2020).

44. Jenkins, B. M. et al. Reflections on the analysis of interfaces and grain boundaries by atom probe tomography. Microsc. Microanal. 26, 247-257 (2020).

45. Zhou, X., Yu, X. X., Kaub, T., Martens, R. L. \& Thompson, G. B. Grain boundary specific segregation in nanocrystalline $\mathrm{Fe}(\mathrm{Cr})$. Sci. Rep. 6, 1-14 (2016).

46. Liebscher, C. H. et al. Strain-Induced Asymmetric Line Segregation at Faceted Si Grain Boundaries. Phys. Rev. Lett. 121, 015702 (2018).

47. Liebscher, C. H. et al. Tetragonal fcc-Fe induced by $\kappa$-Carbide precipitates: Atomic scale insights from correlative electron microscopy, atom probe tomography, and density functional theory. Phys. Rev. Mater. 2, 023804 (2018).

48. Makineni, S. K. et al. On the diffusive phase transformation mechanism assisted by extended dislocations during creep of a single crystal CoNi-Based superalloy. Acta Mater. 155, 362-371 (2018).

49. Stoffers, A. et al. Correlating Atom Probe Tomography with Atomic-Resolved Scanning Transmission Electron Microscopy: Example of Segregation at Silicon Grain Boundaries. Microsc. Microanal. 23, 291-299 (2017). 
50. Herbig, M. Spatially correlated electron microscopy and atom probe tomography: Current possibilities and future perspectives. Scr. Mater. 148, 98-105 (2018).

51. Pennycook, S. J. \& Nellist, P. D. (eds.) Scanning Transmission Electron Microscopy (Springer New York, New York, NY, 2011).

52. Č́a, M., Šob, M. \& Hafner, J. First-principles study of magnetism at grain boundaries in iron and nickel. Phys. Rev. B 78, 054418 (2008).

53. Kubaschewski, O. IRON-Binary Phase Diagrams (Springer Science \& Business Media, 2013).

54. Scheiber, D., Jechtl, T., Svoboda, J., Fischer, F. \& Romaner, L. On solute depletion zones along grain boundaries during segregation. Acta Mater. 182, 100-107 (2020).

55. Lejček, P. \& Hofmann, S. Solute segregation at grain boundaries of a partially ordered Fe3Al base alloy. Surf. Interface Anal. 36, 938-940 (2004).

56. Mintz, B. \& Arrowsmith, J. M. Hot-ductility behaviour of C-Mn-Nb-Al steels and its relationship to crack propagation during the straightening of continuously cast strand. null 6, 24-32 (1979).

57. Scheiber, D., Pippan, R., Puschnig, P. \& Romaner, L. Ab Initio Calculations of Grain boundaries in bcc metals. Model. Simul. Mater. Sci. Eng. 24, 035013 (2016).

58. Scheiber, D., Renk, O., Popov, M. \& Romaner, L. Temperature dependence of surface and grain boundary energies from first principles. Phys. Rev. B 101, 174103 (2020).

59. Schönecker, S., Li, X., Johansson, B., Kwon, S. K. \& Vitos, L. Thermal surface free energy and stress of iron. Sci. Rep. 5, 14860 (2015).

60. White, C. L. \& Coghlan, W. A. The spectrum of binding energies approach to grain boundary segregation. Met. Mat. Trans. A 8, 1403-1412 (1977).

61. Huber, L., Hadian, R., Grabowski, B. \& Neugebauer, J. A machine learning approach to model solute grain boundary segregation. Npj Comput Mater. 4, 1-8 (2018).

62. Fors, D. H. R. \& Wahnström, G. Nature of boron solution and diffusion in $\alpha$-Iron. Phys. Rev. B 77, 132102 (2008).

63. Ophus, C. A fast image simulation algorithm for scanning transmission electron microscopy. Adv. Struct. Chem. Imaging 3, 13 (2017).

64. Pryor, A., Ophus, C. \& Miao, J. A streaming multi-GPU implementation of image simulation algorithms for scanning transmission electron microscopy. Adv. Struct. Chem. Imaging 3, 15 (2017).

65. Aksyonov, D., Hickel, T., Neugebauer, J. \& Lipnitskii, A. The impact of carbon and oxygen in alpha-titanium: Ab initio study of solution enthalpies and grain boundary segregation. J. Phys.: Condens. Matter 28, 385001 (2016).

66. Scheiber, D., Romaner, L., Pippan, R. \& Puschnig, P. Impact of solute-solute interactions on grain boundary segregation and cohesion in molybdenum. Phys. Rev. Mater. 2, 093609 (2018).

67. Scheiber, D., Romaner, L., Fischer, F. \& Svoboda, J. Kinetics of grain boundary segregation in multicomponent systems-The example of a Mo-CBO system. Scr. Mater. 150, 110-114 (2018).

68. Faulkner, R. G. Non-equilibrium grain-boundary segregation in austenitic alloys. J. Mater. Sci. 16, 373-383 (1981).

69. Mehrer, H. \& Stolica, N. Diffusion in solid metals and alloys. vol. 26 (Springer-Verlag, Berlin/Heidelberg, 1990).

70. Erhart, H. \& Grabke, H. J. Equilibrium segregation of phosphorus at grain boundaries of Fe-P, Fe-C-P, Fe-Cr-P, and Fe-Cr-C-P alloys. Met. Sci. 15 , 401-408 (1981).

71. Hristova, E., Janisch, R., Drautz, R. \& Hartmaier, A. Solubility of carbon in $\alpha$ Iron under volumetric strain and close to the $\Sigma 5(310)[001]$ grain boundary: Comparison of DFT and empirical potential methods. Comput. Mater. Sci. 50, 1088-1096 (2011).

72. Hyde, B., Farkas, D. \& Caturla, M. J. Atomistic sliding mechanisms of the $\Sigma=5$ symmetric tilt grain boundary in bcc iron. Philos. Mag. 85, 3795-3807 (2005).

73. Janovec, J., Jenko, M., Lejček, P. \& Pokluda, J. Grain boundary segregation of phosphorus and silicon in polycrystals and bicrystals of the Fe-2.6Si-0.055P alloy. Mater. Sci. Eng. A 462, 441-445 (2007).

74. Yu, Z. et al. Segregation-induced ordered superstructures at general grain boundaries in a nickel-bismuth alloy. Science 358, 97-101 (2017).

75. Kresse, G. \& Hafner, J. Ab initio molecular dynamics for liquid metals. Phys. Rev. B 47, 558 (1993).

76. Kresse, G. \& Furthmüller, J. Efficiency of ab-initio total energy calculations for metals and semiconductors using a plane-wave basis set. Comput. Mater. Sci. 6, 15-50 (1996).
77. Kresse, G. \& Furthmüller, J. Efficient iterative schemes for ab initio totalenergy calculations using a plane-wave basis set. Phys. Rev. B 54, 11169-11186 (1996).

78. Kresse, G. \& Joubert, D. From ultrasoft pseudopotentials to the projector augmented-wave method. Phys. Rev. B 59, 1758-1775 (1999).

79. Perdew, J. P., Burke, K. \& Ernzerhof, M. Generalized gradient approximation made simple. Phys. Rev. Lett. 77, 3865 (1996).

80. Perdew, J., Burke, K. \& Ernzerhof, M. Perdew, Burke, and Ernzerhof reply. Phys. Rev. Lett. 80, 891 (1998).

81. Haas, P., Tran, F. \& Blaha, P. Calculation of the lattice constant of solids with semilocal functionals. Phys. Rev. B 79, 1-10 (2009).

82. Jin, H., Elfimov, I. \& Militzer, M. Study of the interaction of solutes with $\Sigma 5$ (013) tilt grain boundaries in iron using density-functional theory. J. Appl. Phys. 115, 093506 (2014).

\section{Acknowledgements}

We gratefully acknowledge the financial support under the scope of the COMET program within the K2 Center "Integrated Computational Material, Process and Product Engineering (IC-MPPE)" (Project No. 859480). This program is supported by the Austrian Federal Ministries for Transport, Innovation and Technology (BMVIT) and for Digital and Economic Affairs (BMDW), represented by the Austrian research funding association (FFG), and the federal states of Styria, Upper Austria and Tyrol. X.Z. is supported by the Alexander-Humboldt-Stiftung. G.D. gratefully acknowledges support by the ERC Advanced Grant GB-Correlate (Grant Agreement 787446).

\section{Author contributions}

G.D. and L.R. designed the project and secured funding. A.A. conducted the electron microscopy experiments including STEM and FIB/SEM. X.Z. conducted the APT experiments. D.S. performed the simulations. C.L., L.R., and B.G. contributed to the data analysis, C.L. and L.R., as well as G.D., revised the paper.

\section{Funding}

Open Access funding enabled and organized by Projekt DEAL.

\section{Competing interests}

The authors declare no competing interests.

\section{Additional information}

Supplementary information The online version contains supplementary material available at https://doi.org/10.1038/s41467-021-26197-9.

Correspondence and requests for materials should be addressed to A. Ahmadian.

Peer review information Nature Communications thanks the anonymous reviewers for their contribution to the peer review of this work.

Reprints and permission information is available at http://www.nature.com/reprints

Publisher's note Springer Nature remains neutral with regard to jurisdictional claims in published maps and institutional affiliations.

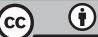

Open Access This article is licensed under a Creative Commons Attribution 4.0 International License, which permits use, sharing, adaptation, distribution and reproduction in any medium or format, as long as you give appropriate credit to the original author(s) and the source, provide a link to the Creative Commons license, and indicate if changes were made. The images or other third party material in this article are included in the article's Creative Commons license, unles indicated otherwise in a credit line to the material. If material is not included in the article's Creative Commons license and your intended use is not permitted by statutory regulation or exceeds the permitted use, you will need to obtain permission directly from the copyright holder. To view a copy of this license, visit http://creativecommons.org/ licenses/by/4.0/.

(c) The Author(s) 2021 